\title{
Celastrol-Induced Suppression of the MiR- 21/ERK Signalling Pathway Attenuates Cardiac Fibrosis and Dysfunction
}

\author{
Mian Cheng ${ }^{a}$ Gang Wu ${ }^{b}$ Yue Song ${ }^{c}$ Lin Wang ${ }^{c}$ Ling Tu $^{a}$ Lizhi Zhang ${ }^{d}$ \\ Cuntai Zhanga
}

aDepartment of Geriatrics, Tongji Hospital, Tongji Medical College, Huazhong University of Science and Technology, Wuhan, China; ${ }^{b}$ Department of Cardiology, Renmin Hospital of Wuhan University, China Cardiovascular Research Institute of Wuhan University, Wuhan, China; 'Department of Cardiology, Tongji Hospital, Tongji Medical College, Huazhong University of Science and Technology, Wuhan, China; dDivision of Anatomic Pathology, Department of Laboratory Medicine and Pathology, Mayo Clinic, Rochester, USA

\section{Key Words}

Micro RNA-21 • Celastrol • ERK • Myocardial fibrosis

\begin{abstract}
Backgroud: Myocardial fibrosis results in myocardial remodelling and dysfunction. Celastrol, a traditional oriental medicine, has been suggested to have cardioprotective effects. However, its underlying mechanism is unknown. This study investigated the ability of celastrol to prevent cardiac fibrosis and dysfunction and explored the underlying mechanisms. Methods: Animal and cell models of cardiac fibrosis were used in this study. Myocardial fibrosis was induced by transverse aortic constriction (TAC) in mice. Cardiac hypertrophy and fibrosis were evaluated based on histological and biochemical measurements. Cardiac function was evaluated by echocardiography. The levels of transforming growth factor beta 1 (TGF- $\beta 1$ ), extracellular signal regulated kinases $1 / 2(E R K 1 / 2)$ signalling were measured using Western blotting, while the expression of miR-21was analyzed by real-time qRT-PCR in vitro and in vivo. In vitro studies, cultured cardiac fibroblasts (CFs) were treated with TGF- $\beta 1$ and transfected with microRNA21(miR21). Results: Celastrol treatment reduced the increased collagen deposition and downregulated $\alpha$-smooth muscle actin ( $\alpha$-SMA), atrial natriuretic peptide (ANP), brain natriuretic peptides (BNP), beta-myosin heavy chain ( $\beta-\mathrm{MHC})$, miR-21 and $p-E R K / E R K$. Cardiac dysfunction was significantly attenuated by celastrol treatment in the TAC mice model. Celastrol treatment reduced myocardial fibroblast viability and collagen content and down-regulated $\alpha-S M A$ in cultured CFs in vitro. Celastrol also inhibited the miR-21/ERK signalling pathway. Celastrol attenuated miR-21 up-regulation by TGF- $\beta 1$ and decreased elevated $p$-ERK/ERK levels in CFs transfected with miR-21. Conclusion: MiR-21/ERK signalling could be a potential therapeutic pathway for the prevention of myocardial fibrosis. Celastrol ameliorates myocardial fibrosis and cardiac dysfunction, these probably related to miR-21/ERK signaling pathways in vitro and in vivo.




\section{Cellular Physiology Cell Physiol Biochem 2016;38:1928-1938

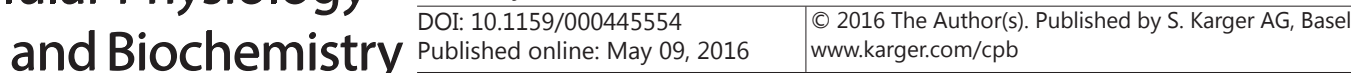 \\ Cheng et al.: Celastrol Ameliorates Cardiac Fibrosis and Dysfunction}

\section{Introduction}

Myocardial fibrosis is a common heart response to many forms of injury and the key pathological process in various cardiovascular diseases. In myocardial fibrosis, excessive collagen deposition and extracellular matrix (ECM) accumulation result in myocardial remodelling, cardiac dysfunction and arrhythmias [1-3]. However, therapeutic strategies for preventing fibrosis remain limited.

MicroRNAs (miRNAs) are non-coding RNAs between 18 and 25 nucleotides in length that are highly conserved. MiRNAs have been shown to participate in biological and pathological processes including cell proliferation, differentiation and apoptosis. Increasing evidence has shown that miRNAs play important roles in the regulation of heart function and structure [4, 5]. MiRNAs exert biological functions by post-transcriptionally regulating gene expression in a sequence-specific manner. The role of miR21 in organ fibrosis has been demonstrated in many studies.

Up-regulation of miR-21 in the heart in response to pressure overload was mainly confined to enhanced expression in cardiac fibroblasts [6], ischemic injury of the heart pathophysiological response. In contrast with the detrimental effects of miR-21 up-regulation in fibroblasts in response to pressure overload, there is evidence that early after myocardial infarction, up-regulation of miR-21 may be protective.

Celastrol is a quinone methide triterpene isolated from the root extracts of Tripterygium wilfordii (thunder god vine) and celastrus regelii [7]. Increasing evidence suggests that celastrol has beneficial effects against tumours, autoimmune and inflammatory diseases $[8,9]$. Celastrol has been demonstrated to induce apoptosis and inhibit cell proliferation in many cancer cells [10]. Der Sarkissian et al. [11] found that celastrol improved cardiac function and inhibited myocardial remodelling in a rat model of myocardial infarction. Kang et al. [12] showed that celastrol suppresses lung cancer invasion and migration by inhibiting TGF- $\beta 1$. TGF- $\beta 1$ is one of the strongest pro-fibrotic factors and is an up-stream signal of miR21 that can induce fibroblast proliferation, differentiation and collagen synthesis [13].

Accordingly, we speculated that celastrol also had significant anti-fibrotic effects in the myocardium and contributed to miR-21 regulation. In this study, we aimed to elucidate the effects of celastrol on myocardial fibrosis and cardiac dysfunction, and explored its potential cellular and molecular mechanisms in in vitro and in vivo models.

\section{Materials and Methods}

Mouse models of transverse aortic constriction

All of the animal experiments were approved by the Animal Experiments Committee of the Tongji Medical College and conformed to the guidelines set forth by the American Association for Laboratory Animal Science (AALAS). Male Kunming mice (20 - 25 g) were provided by the Experimental Animal Centre of Tongji Medical College (Grade II). Mice were housed in an environment at a temperature of $20^{\circ} \mathrm{C}$ with a 12/12 hour night/dark cycle.

Mice were anesthetized with isoflurane and the aorta was exposed, and the transverse aorta was isolated between the right and left carotid arteries. A 7/0 silk ligature using a blunted 27-gauge (0.41-mm OD) needle as a calibrator, ultrasound for the assessment of aortic diameter decreased by $70 \%$. Shamoperated mice underwent the same procedure but without the aortic constriction. Mice received celastrol (1 mg. $\mathrm{kg}^{-1}$ i.p. daily; InvivoGen, San Diego, CA, USA) after TAC. Sham-control and TAC groups were given equal volumes of PBS. We measured the heart weight/body weight (HW/BW) ratio and histological and biochemical measurements in every group 3 weeks after TAC. The cardiac function parameters were evaluated by echocardiography 3 and 12 weeks after TAC.

Isolation and treatment of cardiac fibroblasts

Cardiac tissue samples were dissected, minced, and enzymatically disaggregated by incubation in $0.25 \%$ trypsin and $0.1 \%$ collagenase II for 30 minutes at $37^{\circ} \mathrm{C}$. Myocardiocytes were separated by employing 


\section{Cellular Physiology Cell Physiol Biochem 2016;38:1928-1938 \begin{tabular}{ll|l} 
DOI: 10.1159/000445554 & O 2016 The Author(s). Published by S. Karger AG, Basel \\
www.karger.com/cpb
\end{tabular} \\ Cheng et al.: Celastrol Ameliorates Cardiac Fibrosis and Dysfunction}

differential adhesion. The cardiac fibroblasts were grown in $9.6-\mathrm{cm}^{2}$ six-well cell culture plates with Dulbecco's modified Eagle media (DMEM, HyClone, Logan, UT, USA) containing 10\% foetal bovine serum (FBS, HyClone) at $37^{\circ} \mathrm{C}$ in $5 \% \mathrm{CO}_{2}$ and $95 \%$ air. Twenty percent $\mathrm{FBS}$ cell culture medium was administered to cardiac fibroblasts ( $\mathrm{CFs}$ ) to induce collagen production. Celastrol was dissolved in DMSO. CFs were treated with celastrol at $0.2,1$ and $5 \mu \mathrm{M}$ (final concentration of DMSO was 1\%). Celastrol treatment was started at the same day 2 hours after surgery and given daily for all the period of follow-up. After starvation in serumfree medium for 24 hours, CFs were treated with recombinant human TGF- $\beta 1$ (Sigma-Aldrich Co., LLC, St. Louis, MO, USA) for 24 hours.

\section{Masson's trichrome staining}

The myocardial tissue specimens were fixed in $4 \%$ paraformaldehyde, routinely paraffin embedded, and sliced into $5 \mu \mathrm{m}$ thick sections. The paraffin sections were quickly dissected and immersed in $10 \%$ neutral buffered formalin and stained with Masson's Trichrome (Accustain HT15; Sigma-Aldrich, St. Louis, MO, USA) for 24 hours to detect the fibrotic areas. Fibrosis tissue was quantified with image analysis software (Image-Pro Plus v4.0; Media Cybernetics, Bethesda, MD, USA). The collagen volume fraction was calculated as the mean ratio of the connective tissue to the total tissue area from all of the measurements from the section, omitting fibrosis from the perivascular, epicardial and endocardial areas.

\section{Echocardiographic assessment}

At 3 and 12 weeks, each mouse was anesthetized with $1 \%$ inhaled isoflurane and set in a supine position. Echocardiographic assessments of the left ventricular (LV) anatomy and its function were performed using a Sonos 5500 Imaging System (Philips, Philips Healthcare, Andover, MA, USA) with a 12 $\mathrm{MHz}$ transducer. The left ventricle anterior wall during diastole (LVAWD), left ventricle anterior wall during systole (LVAWS), left ventricle posterior wall during diastole (LVPWD), left ventricle posterior wall during systole (LVPWS), left ventricle mass (LV mass), ejection fraction (EF) and fractional shortening (FS) were measured. Echocardiographic measurements were averaged from at least three separate cardiac cycles.

\section{Transfection procedure}

For transfection, 24 hours before any experiment cells were plated onto 6-well plates and the confluence is up to $60-80 \%$, incubated with serum-free medium. $5 \mu \mathrm{L}$ of the miRNA $(20 \mu \mathrm{M})$ and Lipofectamine 2000 (Invitrogen, Carlsbad, CA, USA) were separately mixed with $250 \mu \mathrm{L}$ of Opti-MEM ${ }^{\circledR}$ Reduced Serum Medium (Gibco, Grand Island, NY, USA) for 5 mins, and then the Lipofectamine-miRNA (Ratio 1:1.5) mixtures were combined and incubated for 20 mins. Subsequently, the mixture was added to each well and incubated at $37^{\circ} \mathrm{C}$ for 6 hours. After that, washing twice with $1 \%$ PBS, and $2 \mathrm{~mL}$ fresh medium containing $10 \%$ FBS was added to the plate, respectively. Then the cells were maintained in culture before other experiments were performed.

\section{Cell viability measurements}

Primary CFs were treated with celastrol $(0.2,1$ and $5 \mu \mathrm{M})$ for 24,48 and 72 hours. Cells were seeded into a 96-well plate at a density of $1 \times 10^{4}$ cells/well and allowed to adhere overnight. Cells were incubated with $10 \mu \mathrm{L}$ 3-(4, 5-dimethylthiazol-2-yl)-2, 5-diphenyl-2H-tetrazolium bromide (MTT) solution for 4 hours at $37^{\circ} \mathrm{C}$ in the dark. Each well then received $150 \mu \mathrm{L}$ of DMSO. The absorbance was measured using a Universal Microplate Spectrophotometer (Tecan, Grödig, Austria) at $570 \mathrm{~nm}$.

\section{Measurement of collagen content}

Total collagen content was measured by the Sircol Collagen Assay Kit (Biocolor, Belfast, Northern Ireland) according to the manufacturer's protocol. Each sample was treated with $50 \mu \mathrm{L}$ collagen lysate and allowed to incubate for 24 hours at $4^{\circ} \mathrm{C}$. After centrifugation, each supernatant was assayed at $562 \mathrm{~nm}$ with a BCA protein assay kit to determine total collagen content. Sircol dye reagent, which binds to collagen (300 $\mu \mathrm{L}$ ), was added to each sample and then mixed for 30 minutes at $4^{\circ} \mathrm{C}$. After centrifugation, the sediment was suspended in $500 \mu \mathrm{L}$ of an alkali reagent to dissolve the bound dye and the absorbance value was measured at $540 \mathrm{~nm}$ with a spectrophotometer. Readings were converted to protein units using a linear calibration curve generated from standards (Vitrogen 100; Angiotech Biomaterials, Palo Alto, CA, USA) and normalized to the total protein of each sample $(1.2-1.5 \mathrm{mg})$. 


\section{Cellular Physiology Cell Physiol Biochem 2016;38:1928-1938 \\ \begin{tabular}{l|l} 
and Biochemistry Published online: May 09, 2016 & $\begin{array}{l}\text { (c) 2016 The Author(s). Published by S. Karger AG, Basel } \\
\text { www.karger.com/cpb }\end{array}$
\end{tabular} \\ Cheng et al.: Celastrol Ameliorates Cardiac Fibrosis and Dysfunction}

Quantitative real-time RT-PCR

Total RNA was extracted from the cultured cells using the Trizol reagent (Life Technologies, Rockville, MD, USA). The amount of RNA was assessed spectrophotometrically using a NanoDrop ND-1000 (NanoDrop Technologies, Wilmington, DE, USA). Extracted RNA was reverse-transcribed into cDNA using the PrimeScript RT reagent kit (Takara Bio, Dalian, China) according to the manufacturer's protocol. Quantitative real-time RT-PCR was performed on an ABI PRISM 7900 real-time PCR system (Applied Biosystems, Foster City, CA, USA). The following primers were used during reverse transcription and PCR: (1) Mmu-miR-21 RT primer, 5'-GTC GTA TCC AGT GCG TGT CGT GGA GTC GGC AAT TGC ACT GGA TAC GAC TCA ACATC-3'; sense primer, 5'-GGG GTA GCT TAT CAG ACT GATG-3' and antisense primer, 5'TGT CGT GGA GTC GGC AATTG-3' and (2) U6 RT primer, 5'-CGCT TCA CGA ATT TGC GTG TCAT-3'; sense primer, 5'-GCT TCG GCA GCA CAT ATA CTA AAAT3' and antisense primer, 5'-CGC TTC ACG AAT TTG CGT GTCAT-3'.

\section{Western blotting}

Total protein was extracted from cells and its concentration was determined using a bicinchoninic acid kit. The samples were lysed with RIPA lysis buffer (Beyotime, Jiangsu, China). Proteins were separated on 15\% SDS-polyacrylamide gels. After electrophoresis, the proteins were transferred to pure nitrocellulose membranes (Biotrace NT, Pall, FL, USA) and blocked for 2 hours with 5\% dried milk in Tris-buffered saline. The membranes were then incubated with the following primary antibodies: (1) anti- $\alpha$-SMA (1:2000 dilutions, mouse monoclonal; Abcam Inc., Cambridge, MA, USA) and (2) anti-ERK1/2 and antiphosphoERK1/2 (1:10,000 dilutions, rabbit polyclonal; Cell Signaling Technology, Beverly, MA, USA). Fluorescent anti-rabbit secondary antibodies were used for detection and the blots were examined using an Odyssey Infrared Imaging System (Gene Company Limited, Hong Kong, China).

Statistical assays

All of the data are presented as the mean \pm SEM. Differences were evaluated by one-way ANOVA with a P-value $<0.05$ being considered significant. Data were analysed using GraphPad Prism 5.0 and SPSS 14.0.

\section{Results}

Celastrol attenuates mouse cardiac hypertrophy and improves cardiac function induced by TAC

To detect the cardioprotective effects of celastrol on pathological myocardial hypertrophy and dysfunction, we treated TAC-operated mice with celastrol or vehicle (PBS). HB/BW, ANP, BNP and $\beta$-MHC were determined after 3 weeks. LVAWD, LVAWS, LVPWD, LVPWS and LV mass were measured by echocardiography after 3 weeks and EF and FS were measured after 12 weeks.

TAC induced substantial left ventricular remodelling and increased the cardiac mass when compared with the control. Celastrol treatment attenuated the increase in cardiac mass (Fig. 1A). TAC significantly increased HW/BW compared with the sham-operated group. Celastrol treatment attenuated the increase in HW/BW (Fig. 1B). Echocardiography examination showed that LVAWD, LVAWS, LVPWD, LVPWS and LV mass significantly increased in the TAC model after 3 weeks and that EF and FS decreased after 12 weeks compared with the sham-operated group. Celastrol treatment attenuated left ventricular hypertrophy and dysfunction compared with the TAC group (Fig. 1C, Table 1). Reactivation of foetal genes are reliable markers for cardiac hypertrophy and heart failure and play important roles in cardiac remodeling, including ANF, BNP, and $\beta$-MHC. TAC significantly up-regulated the expression of these mRNAs compared with the sham-operated group. Celastrol treatment attenuated the upregulation of these markers (Fig. 1D). These results suggest that celastrol significantly attenuates TAC-induced myocardial hypertrophy and dysfunction.

Celastrol attenuates mouse pathological myocardial fibrosis induced by TAC

After 3 weeks, Masson staining showed that TAC induced massive collagen production. Celastrol treatments alleviated TAC induced collagen deposition (Fig. 2A and B). In the 


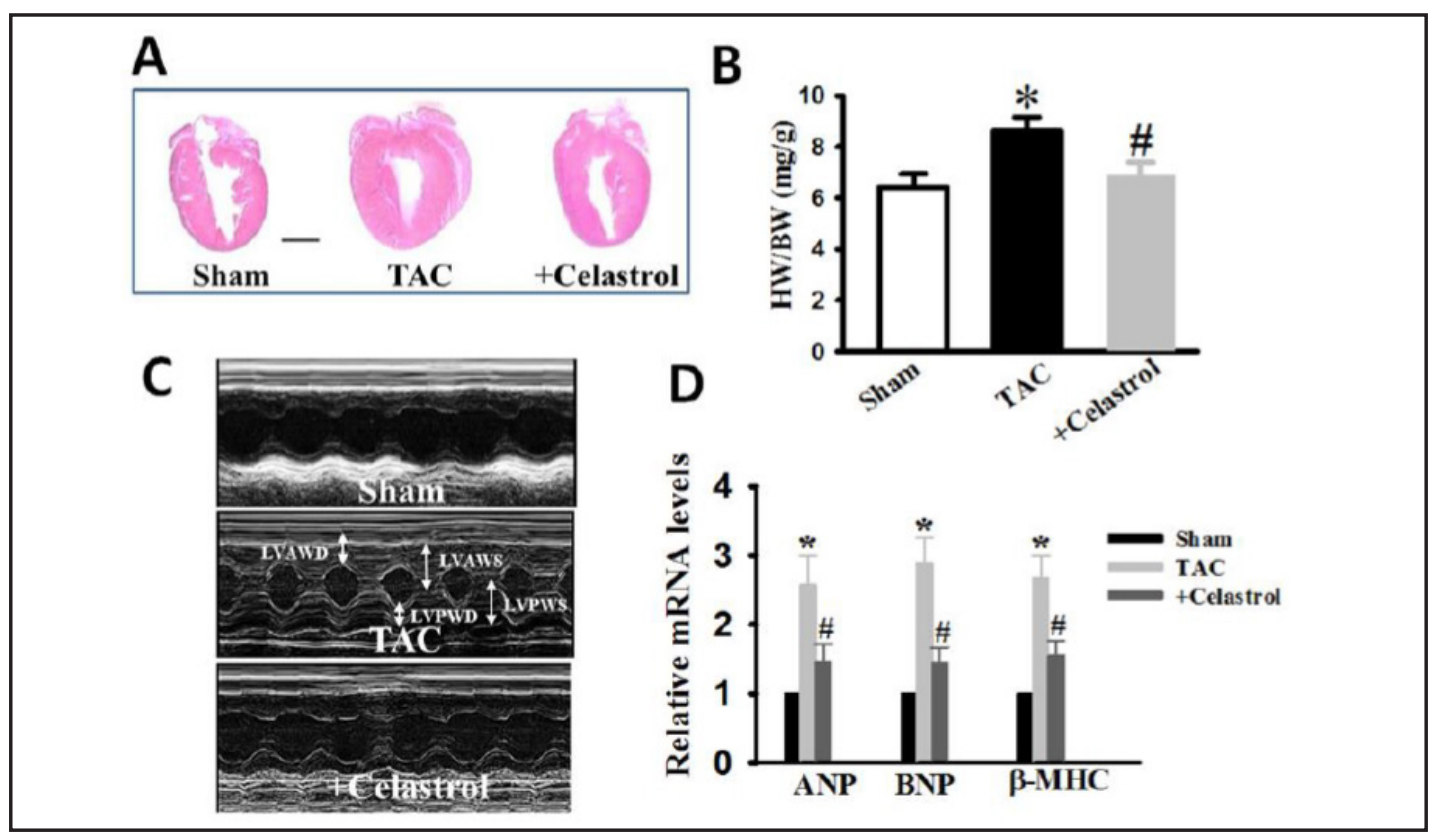

Fig. 1. Celastrol attenuates cardiac hypertrophy and improves cardiac function in TAC mice. (A) Representative histological heart sections from each group. Scale $=1 \mathrm{~mm}$. (B) Heart weight was normalized to body weight (HW/BW, mg/g). HW/BW was increased in the TAC group compared with the control group and decreased in TAC+celastrol group. (C) LVAWD, LVAWS, LVPWD, LVPWS, EF and FS were measured by echocardiography. (D) ANP, BNP and $\beta$-MHC gene expression increased in the TAC group compared with the control group and decreased in the TAC+celastrol group. Data are shown as the mean \pm SEM. $* \mathrm{P}<0.05$ versus the control group and $\# \mathrm{P}<0.05$ versus the TAC group. $\mathrm{n}=6$ for each group.

sham-operated groups, the myocardium demonstrated orderly myofibril distribution; collagen was rarely observed in the myocardial interstitial. In the TAC groups, cardiomyocytes presented fractured mitochondrial cristae, disorderly sarcomere structures and an interstitial space filled with fasciculate collagen. In the celastrol-treated group, less collagen deposition was observed than TAC group. $\alpha$-SMA was up-regulated in TAC myocardial tissue; celastrol treatment down-regulated $\alpha$-SMA expression (Fig. 2C and D). These results suggest that celastrol significantly prevents the development of myocardial fibrosis in vivo.
Table 1. Echocardiography parameters at baseline, after TAC treatment and after celastrol treatment for 3 and 12 weeks. $* \mathrm{P}<0.05$ versus the control group and \# $\mathrm{P}<0.05$ versus the TAC group, $(\mathrm{n}=6)$

\begin{tabular}{lccl}
\hline $\begin{array}{l}\text { Group } \\
\text { 3weeks }\end{array}$ & $\begin{array}{c}\text { Sham } \\
(n=8) \\
\text { 12weeks }\end{array}$ & $\begin{array}{c}\text { TAC } \\
(n=8) \\
(n=8)\end{array}$ & $\begin{array}{c}\text { +Celastrol } \\
(n=8) \\
(n=8)\end{array}$ \\
\hline LVAWD (mm) & $0.77 \pm 0.11$ & $1.45 \pm 0.13^{*}$ & $0.98 \pm 0.16 \#$ \\
LVAWS (mm) & $1.27 \pm 0.18$ & $2.16 \pm 0.16^{*}$ & $1.61 \pm 0.24 \#$ \\
LVPWD (mm) & $0.75 \pm 0.12$ & $1.48 \pm 0.20^{*}$ & $0.97 \pm 0.14 \#$ \\
LVPWS (mm) & $1.19 \pm 0.15$ & $2.01 \pm 0.18^{*}$ & $1.45 \pm 0.29 \#$ \\
LV mass (mg) & $70.22 \pm 6.57$ & $172.47 \pm 25.22^{*}$ & $116.19 \pm 18.09 \#$ \\
EF (\%) 12weeks & $65.57 \pm 6.49$ & $33.32 \pm 6.49^{*}$ & $43.69 \pm 6.11 \#$ \\
FS (\%) 12weeks & $36.61 \pm 5.21$ & $16.08 \pm 4.28^{*}$ & $25.36 \pm 3.43 \#$ \\
\hline
\end{tabular}

Celastrol inhibits miR-21 expression and ERK1/2 phosphorylation in vivo

To examine whether miR-21 participates in myocardial remodelling in a TAC mouse model and whether it can be regulated by celastrol treatment, miR-21 levels and ERK1/2 total protein levels and phosphorylation states were detected. The results showed that miR-21 was up-regulated in the TAC model compared with the sham-operated group; these changes were significantly attenuated by celastrol (Fig. 3A). Western blot analysis revealed that ERK1/2 phosphorylation is significantly increased in the TAC model and abolished with celastrol treatment (Fig. 3B and C). 


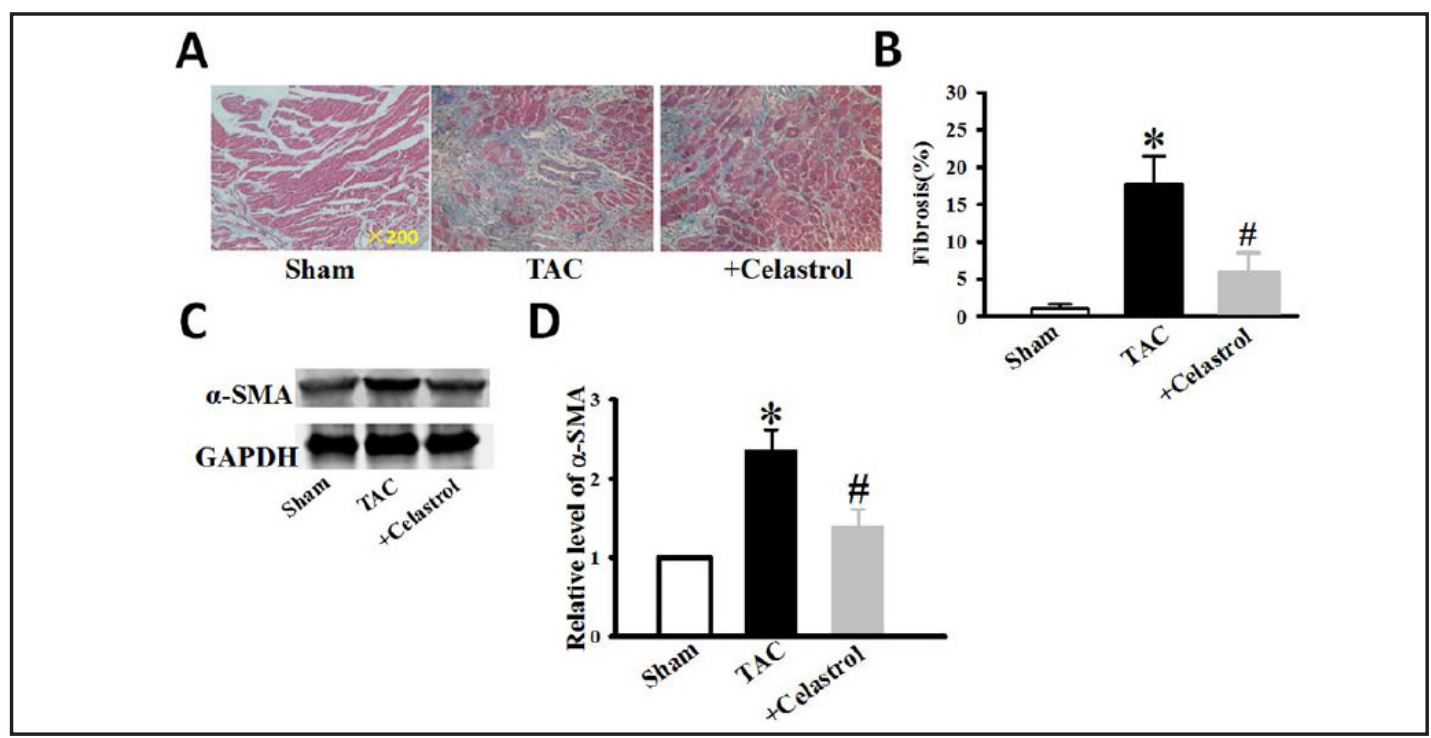

Fig. 2. Celastrol reduces pathological myocardial fibrosis in mice caused by TAC $\left(1 \mathrm{mg} \cdot \mathrm{kg}^{-1}\right.$ i.p. daily for 3 weeks after TAC). (A) Masson's trichrome-stained sections from myocardial tissue showed that collagen deposition was significantly reduced in the TAC + celastrol group compared with the TAC group. Scale bar = $100 \mu \mathrm{m}$. (B) Quantification of cardiac fibrosis areas from Masson's trichrome-stained sections. The images of each group were analysed using the Image Pro Plus software. (C) Western blotting was used to determine $\alpha$-SMA expression. $\alpha$-SMA was up-regulated in TAC myocardial tissue. In contrast, celastrol treatment down-regulated $\alpha$-SMA expression. (D) The intensity of each band shown in (C) was quantified by densitometry and the data were normalized to the GAPDH protein band intensity. $\alpha$-SMA expression was up-regulated in the TAC myocardial tissue and down-regulated following celastrol treatment. Data are shown as the mean \pm SEM. $* \mathrm{P}<0.05$ versus the control group and $\# \mathrm{P}<0.05$ versus the TAC group. $\mathrm{n}=6$ for each group.

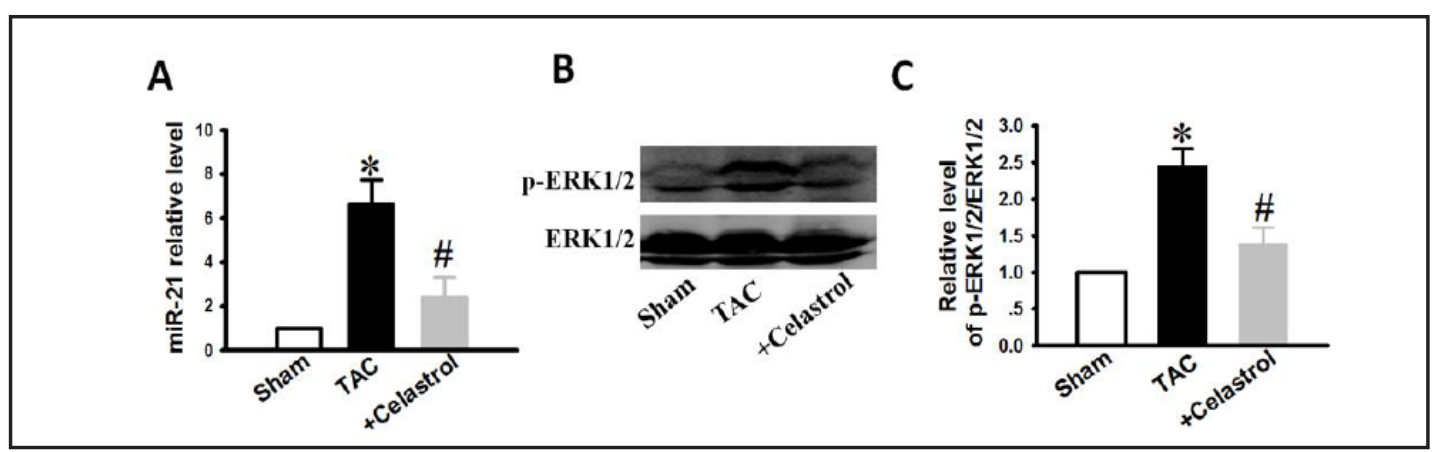

Fig. 3. Celastrol inhibits miR-21 expression and ERK1/2 phosphorylation induced by TAC. Mice were treated with celastrol (1 mg-kg-1 i.p. daily) for 3 weeks after TAC. (A) TAC increased miR-21 expression, which was abolished by celastrol treatment. (B) Western blotting was used to determine p-ERK1/2 and ERK1/2 expression. TAC promoted ERK1/2 phosphorylation, which was abolished by celastrol treatment. (C) The intensity of each band shown in (B) was quantified by densitometry. The densitometry analysis of ERK activation as a ratio of p-ERK to total ERK1/2. TAC promoted ERK1/2 phosphorylation, which was abolished by celastrol treatment. Data are shown as the mean \pm SEM. $n=6$ for each group. ${ }^{*} \mathrm{P}<0.05$ versus the control group and \# $\mathrm{P}<0.05$ versus the TAC group.

Effect of celastrol on viability in cardiac fibroblasts and collagen content in neonatal cardiac fibroblasts

Several studies have reported that celastrol decreases cell viability in various cell types $[14,15]$. Fibroblast is a principal characteristic in cardiac fibrosis. Cardiac fibroblasts synthesize ECM and may directly cause cardiomyocyte hypertrophy via paracrine mechanisms, which further contribute to impaired cardiac function [16]. Therefore, we KARGER 
Fig. 4. Celastrol decreases $\mathrm{CF}$ viability and collagen content. (A) Time- and dose-dependent effect of celastrol on the inhibition of CF viability as measured by MTT assay. (B) CF collagen content increased after treatment with $20 \%$ foetal bovine serum (FBS) and decreased after $0.2,1$ and $5 \mu \mathrm{M}$ celastrol treatment. (C) Celastrol treatment abolished FBS-induced expression of $\alpha$-SMA in a dose-dependent manner. (D) The intensity of each band shown in (C) was quantified by densitometry. Celastrol treatment abolished FBS-induced expression of $\alpha$-SMA in a dose-dependent manner. Data

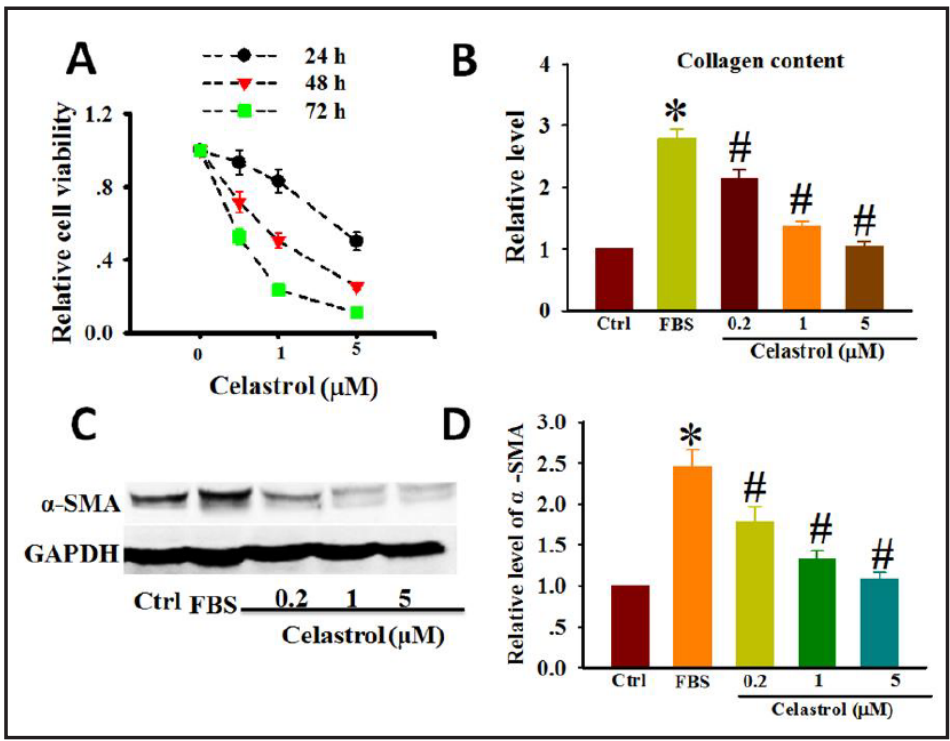
are shown as the mean \pm SEM. $* \mathrm{P}<0.05$ versus the control group and $\# \mathrm{P}<0.05$ versus the FBS-treated cells.

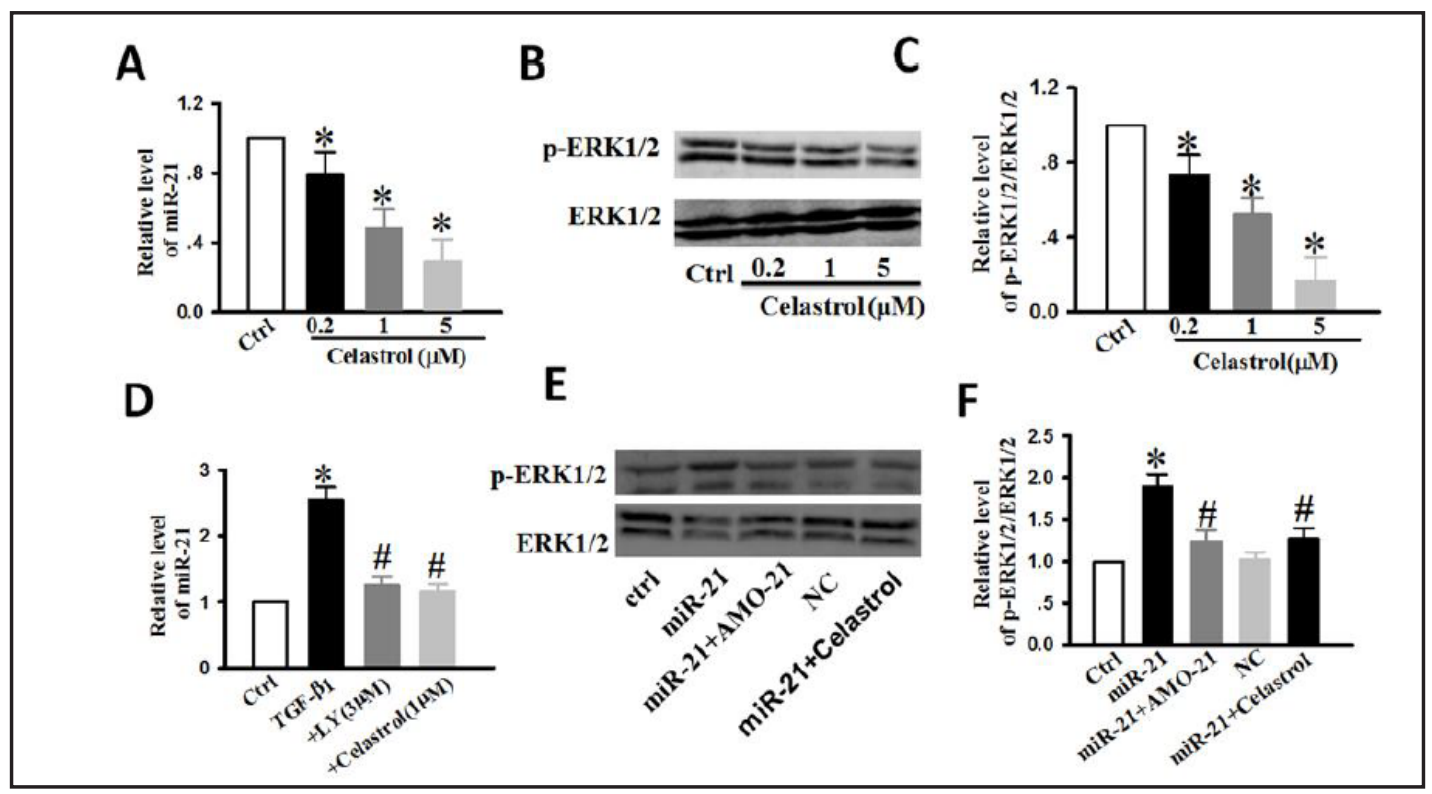

Fig. 5. Celastrol inhibits miR-21 expression and its downstream MAPK/ERK signals in CFs. (A) Bar diagram summarizing the inhibitory effect of celastrol on miR-21 expression in a dose-dependent manner in CFs ( $\mathrm{n}$ = 3). ${ }^{*} \mathrm{P}<0.05$. (B) pERK1/2 protein levels were decreased after $0.2,1$ and $5 \mu \mathrm{M}$ celastrol treatment in CFs $(n=3) .(C)$ The intensity of each band shown in (B) was quantified by densitometry. The densitometry analysis of ERK activation as a ratio of p-ERK to total ERK1/2. Celastrol alleviated the increase in p-ERK/ERK in CFs ( $n=3$ ). Columns, mean; bars, SEM * $\mathrm{P}<0.05$. (D) miR-21 expression was increased in CFs treated with TGF- $\beta 1$ and decreased following LY2109761 or celastrol treatment $(n=3)$. LY2109761 is a TGF- $\beta 1$ inhibitor. Columns, mean; bars, SEM *P $<0.05$ versus the control group and \# $\mathrm{P}<0.05$ versus the TGF- $\beta 1$-treated cells. (E) Transfection of miR-21 into CFs resulted in ERK1/2 phosphorylation compared with the control. Celastrol and AMO-21, an inhibitor of endogenous miR-21, alleviated the increase in p-ERK/ERK $(n=3)$. (F) The intensity of each band shown in (E) was quantified by densitometry. The densitometry analysis of ERK activation as a ratio of p-ERK to total ERK1/2. Celastrol alleviated the increase in p-ERK/ERK in CFs transfected with miR-21 ( $\mathrm{n}=3$ ). Data are shown as the mean \pm SEM. $* \mathrm{P}<0.05$ versus the control group and $\# \mathrm{P}<0.05$ versus the TGF- $\beta 1$ group. 


\section{Cellular Physiology Cell Physiol Biochem 2016;38:1928-1938 \\ \begin{tabular}{ll|l} 
DOI: 10.1159/000445554 & O 2016 The Author(s). Published by S. Karger AG, Basel \\
www.karger.com/cpb
\end{tabular} \\ Cheng et al.: Celastrol Ameliorates Cardiac Fibrosis and Dysfunction}

measured CF viability via MTT assay and tested the effect of celastrol on CF viability. FBS promoted fibroblast viablity, whereas celastrol eliminated this effect. CFs were treated with varying celastrol concentrations $(0.2,1$ and $5 \mu \mathrm{M})$ for 24,48 and 72 hours. Cell viability was determined using the MTT assay (Fig. 4A). The results confirmed that celastrol inhibits fibroblast viability in a time- and dose-dependent manner.

The primary CFs were stimulated with 20\% FBS for 24 hours after pre-treatment with celastrol $(0.2,1$ and $5 \mu \mathrm{M})$ for 1 hour. As shown in Fig. 4B, primary CF collagen content increased after treatment with FBS and was significantly attenuated by celastrol pre-treatment at 0.2, 1 and $5 \mu \mathrm{M}$. Celastrol pre-treatment largely abolished FBS-induced expression of $\alpha$-SMA (Fig. 4C and D).

Celastrol inhibits miR-21/ERK activation in neonatal cardiac fibroblasts

MiR-21 plays a crucial role in fibrosis [17]. Previous results suggest that miR-21 and p-ERK/ERK are up-regulated in the TAC model. The effect of celastrol on fibroblast viability indicate that miR-21/ERK activation may be involved in the signalling pathway that underlies celastrol's anti-fibrotic actions. CFs were treated with celastrol $(0.2,1$ and $5 \mu \mathrm{M})$ for 1 hour. MiR-21 and p-ERK/ERK expression were down-regulated in a dose-dependent manner. (Fig. $5 A, B$ and C) TGF- $\beta 1$ is a miR-21 upstream signal. Thus, we detected miR-21 levels in TGF$\beta 1$-treated CFs. TGF- $\beta 1$ treatment up-regulated miR-21 compared with the control group. A TGF- $\beta 1$ inhibitor (LY2109761) and celastrol suppressed the expression of miR-21 originally induced by TGF- $\beta 1$. (Fig. 5D).

Celastrol inhibited ERK1/2 phosphorylation in vivo and in vitro. Thus, we investigated whether the inhibition of ERK1/2 activity caused by celastrol was through miR-21 inhibition. We found that miR-21 overexpression up-regulated p-ERK/ERK levels compared with the negative control. Notably, celastrol decreased p-ERK/ERK levels in CFs transfected with miR-21. AMO-21 is an endogenous miR-21 inhibitor. Co-application of miR-21 with AMO-21 alleviated the increase in p-ERK/ERK (Fig. 5E and F).

\section{Discussion}

The present study showed that celastrol was able to suppress pathological myocardial fibrosis and prevent cardiac dysfunction. Celastrol markedly reduced collagen deposition and improved cardiac function in a TAC model, inhibited CF viability in a time- and dosedependent manner and suppressed collagen content by inhibiting the miR-21/ERK signalling pathway both in vitro and in vivo.

Cardiac fibrosis is a common reactive response in many cardiac pathologic conditions that results in cardiac structural and functional alterations. Celastrol suppresses pathological myocardial fibrosis in a mouse TAC model. At the initial phase of pressure overload, the heart exhibits an adaptive response, followed by cardiac hypertrophy, fibroblast proliferation and increased collagen and ECM deposition [18]. In the current study, Masson staining showed a significant increase in collagen deposition, left ventricular wall thickening as measured by echocardiography and increased expression of foetal genes after 3 weeks of TAC. As fibrosis progresses, cardiac dysfunction occurs $[19,20]$. After 12 weeks, long-standing pressure overload resulted in cardiac dysfunction. Moreover, celastrol significantly prevented cardiac fibrosis and dysfunction. We found that celastrol reverses cardiac fibrosis, downregulates miR-21 caused by pressure overload and attenuates the activated ERK1/2 signalling pathway. MiR-21 plays a crucial role in cardiac fibrosis and is predominantly expressed in cardiac fibroblasts $[6,21]$. miR-21 has been shown to promote fibroblast survival, which contributes to myocardial fibrosis [22]. In cardiac fibrosis related to AngII, miR-21 was transcriptionally activated and increased fibroblast survival [23]. Our investigation showed that celastrol inhibited miR-21 up-regulation both in vitro and in vivo and decreased CF viability, suggesting a potential role for miR-21 in cardiac fibrosis progression. MiR-21 overexpression led to a significant increase in MAPK/ERK activation. AMO-21 (an inhibitor 


\section{Cellular Physiology Cell Physiol Biochem 2016;38:1928-1938 \begin{tabular}{c|c|c|} 
DOI: 10.1159/000445554 & O 2016 The Author(s). Published by S. Karger AG, Basel \\
www.karger.com/cpb
\end{tabular} \\ Cheng et al.: Celastrol Ameliorates Cardiac Fibrosis and Dysfunction}

of endogenous miR-21) alleviated this increase in p-ERK/ERK. Studies have also shown that miR-21 inhibits the apoptotic response in cardiac fibroblasts through MAPK/ERK signalling. Thum et al. [6] showed that miR-21 up-regulates the MAPK/ERK signalling pathway in cardiac fibroblasts, which has impacts on global cardiac structure and function. Moreover, miR-21 silencing reduced cardiac ERK-MAP kinase activity, inhibited interstitial fibrosis and attenuated cardiac dysfunction.

In our study, CFs exposed to TGF- $\beta 1$ display high miR-21 expression levels. Celastrol as well as LY2109761 (an inhibitor of TGF- $\beta 1$ ) reduce the increased miR-21 expression induced by TGF- $\beta 1$. Furthermore, studies have shown that TGF- $\beta 1$ downregulation can also reduce cardiac fibrosis [24]. TGF- $\beta 1$ is considered to be a key molecule in the activation of the fibrotic programme. TGF- $\beta 1$ induces fibroblast conversion into myofibroblasts and enhances extracellular matrix protein synthesis [25]. Zeisberg et al. reported that TGF- $\beta 1$ induces endothelial cells to undergo an endothelial-mesenchymal transition [26]. Additionally, TGF- $\beta 1$ suppresses the activity of proteases that degrade the extracellular matrix by inhibiting matrix metallo proteinase (MMP) expression and inducing the synthesis of protease inhibitors [27]. TGF- $\beta 1$ is an upstream positive regulator of miR-21, and research shows that miR-21 up-regulation by TGF- $\beta 1$ promotes cardiac fibrosis [28]. These results suggest that celastrol inhibits TGF- $\beta 1 / \mathrm{miR}-21$ signalling to prevent cardiac fibrosis. In myocardial infarction, miR-21 activates the TGF- $\beta 1 /$ Smad pathway via suppression of the TGF- $\beta$ receptor III in ischaemic areas, enhancing collagen production, up-regulating $\alpha$-SMA expression and facilitating fibroblast differentiation into pathological myofibroblasts [29]. In conclusion, the inhibitory mechanisms of cardiac fibrosis caused by celastrol were associated with miR-21/ ERK down-regulation; this effect occurred along with downregulation of the TGF- $\beta 1$ signalling pathway. These results aid in our understanding of cardiac fibrosis mechanisms, which are reduced by celastrol in vitro and in vivo.

Our study demonstrated for the first time that celastrol inhibits myocardial fibroblast proliferation and fibrosis by downregulating miR-21 expression and inhibiting MAPK/ERK signalling. These data show that celastrol is a promising new agent for preventing myocardial fibrosis, improving cardiac function and even reversing ventricular remodelling.

\section{Acknowlegments}

This work was supported by the national natural science foundation of China (No: 81270305).

\section{Disclosure Statement}

None of the authors had any conflicts of interest and/or financial disclosures to disclose.

\section{References}

1 Broberg CS, Burchill LJ: Myocardial factor revisited: The importance of myocardial fibrosis in adults with congenital heart disease. Int J Cardiol 2015;189:204-210.

2 Donekal S, Venkatesh BA, Liu YC, Liu CY, Yoneyama K, Wu CO, Nacif M, Gomes AS, Hundley WG, Bluemke DA, Lima JA: Interstitial fibrosis, left ventricular remodeling, and myocardial mechanical behavior in a population-based multiethnic cohort: the Multi-Ethnic Study of Atherosclerosis (MESA) study. Circ Cardiovasc Imaging 2014;7:292-302.

3 Lorell BH, Carabello BA: Left ventricular hypertrophy: pathogenesis, detection, and prognosis. Circulation 2000;102:470-479. 


\section{Cellular Physiology Cell Physiol Biochem 2016;38:1928-1938 \begin{tabular}{c|c|c|} 
DOI: 10.1159/000445554 & $\begin{array}{l}\text { O 2016 The Author(s). Published by S. Karger AG, Basel } \\
\text { www.karger.com/cpb }\end{array}$
\end{tabular} \\ Cheng et al.: Celastrol Ameliorates Cardiac Fibrosis and Dysfunction}

4 Melo SF, Fernandes T, Barauna VG, Matos KC, Santos AA, Tucci PJ, Oliveira EM: Expression of MicroRNA-29 and Collagen in Cardiac Muscle after Swimming Training in Myocardial-Infarcted Rats. Cell Physiol Biochem 2014;33:657-669.

5 Zhao X, Wang K, Liao Y, Zeng Q, Li Y, Hu F, Liu Y, Meng K, Qian C, Zhang Q Guan H, Feng K, Zhou Y, Du Y, Chen Z: MicroRNA-101a inhibits cardiac fibrosis induced by hypoxia via targeting TGFbetaRI on cardiac fibroblasts. Cell Physiol Biochem 2015;35:213-226.

6 Thum T, Gross C, Fiedler J, Fischer T, Kissler S, Bussen M, Galuppo P, Just S, Rottbauer W, Frantz S, Castoldi M, Soutschek J, Koteliansky V, Rosenwald A, Basson MA, Licht JD, Pena JT, Rouhanifard SH, Muckenthaler MU, Tuschl T, Martin GR, Bauersachs J, Engelhardt S: MicroRNA-21 contributes to myocardial disease by stimulating MAP kinase signalling in fibroblasts. Nature 2008;456:980-984.

7 Salminen A, Lehtonen M, Paimela T, Kaarniranta K: Celastrol: Molecular targets of Thunder God Vine. Biochem Biophys Res Commun 2010;394:439-442.

8 Jung HW, Chung YS, Kim YS, Park YK: Celastrol inhibits production of nitric oxide and proinflammatory cytokines through MAPK signal transduction and NF-kappaB in LPS-stimulated BV-2 microglial cells. Exp Mol Med 2007;39:715-721.

9 Kim DY, Park JW, Jeoung D, Ro JY: Celastrol suppresses allergen-induced airway inflammation in a mouse allergic asthma model. Eur J Pharmacol 2009;612:98-105.

10 Li PP, He W, Yuan PF, Song SS, Lu JT, Wei W: Celastrol induces mitochondria-mediated apoptosis in hepatocellular carcinoma Bel-7402 cells. Am J Chin Med 2015;43:137-148.

11 Der Sarkissian S, Cailhier JF, Borie M, Stevens LM, Gaboury L, Mansour S, Hamet P, Noiseux N: Celastrol protects ischaemic myocardium through a heat shock response with up-regulation of haeme oxygenase-1. Br J Pharmacol 2014;171:5265-5279.

12 Kang H, Lee M, Jang SW: Celastrol inhibits TGF-beta1-induced epithelial-mesenchymal transition by inhibiting Snail and regulating E-cadherin expression. Biochem Biophys Res Commun 2013;437:550-556.

13 Sheppard D: Transforming growth factor beta: a central modulator of pulmonary and airway inflammation and fibrosis. Proc Am Thorac Soc 2006;3:413-417.

14 Shrivastava S, Jeengar MK, Reddy VS, Reddy GB, Naidu VG: Anticancer effect of celastrol on human triple negative breast cancer: possible involvement of oxidative stress, mitochondrial dysfunction, apoptosis and PI3K/Akt pathways. Exp Mol Pathol 2015;98:313-327.

15 Ji N, Li J, Wei Z, Kong F, Jin H, Chen X, Li Y, Deng Y: Effect of celastrol on growth inhibition of prostate cancer cells through the regulation of hERG channel in vitro. Biomed Res Int 2015;2015:308475.

16 Jiang ZS, Jeyaraman M, Wen GB, Fandrich RR, Dixon IM, Cattini PA, Kardami E: High- but not low-molecular weight FGF-2 causes cardiac hypertrophy in vivo; possible involvement of cardiotrophin-1. J Mol Cell Cardiol 2007;42:222-233.

17 Kim EK, Choi E-J: Pathological roles of MAPK signaling pathways in human diseases. Biochim Biophys Acta 2010;1802:396-405.

18 Petrov VV, Fagard RH, Lijnen PJ: Stimulation of collagen production by transforming growth factor-beta1 during differentiation of cardiac fibroblasts to myofibroblasts. Hypertension 2002;39:258-263.

19 Thum T, Galuppo P, Wolf C, Fiedler J, Kneitz S, van Laake LW, Doevendans PA, Mummery CL, Borlak J, Haverich A, Gross C, Engelhardt S, Ertl G, Bauersachs J: MicroRNAs in the human heart: a clue to fetal gene reprogramming in heart failure. Circulation 2007;116:258-267.

20 Janicki JS, Brower GL: The role of myocardial fibrillar collagen in ventricular remodeling and function. J Card Fail 2002;8:S319-325.

21 Roy S, Khanna S, Hussain SR, Biswas S, Azad A, Rink C, Gnyawali S, Shilo S, Nuovo GJ, Sen CK: MicroRNA expression in response to murine myocardial infarction: miR-21 regulates fibroblast metalloprotease-2 via phosphatase and tensin homologue. Cardiovasc Res 2009;82:21-29.

22 Chan JA, Krichevsky AM, Kosik KS: MicroRNA-21 is an antiapoptotic factor in human glioblastoma cells. Cancer Res 2005;65:6029-6033.

23 Lorenzen JM, Schauerte C, Hubner A, Kolling M, Martino F, Scherf K, Batkai S, Zimmer K, Foinquinos A, Kaucsar T, Fiedler J, Kumarswamy R, Bang C, Hartmann D, Gupta SK, Kielstein J, Jungmann A, Katus HA, Weidemann F, Muller OJ, Haller H, Thum T: Osteopontin is indispensible for AP1-mediated angiotensin IIrelated miR-21 transcription during cardiac fibrosis. Eur Heart J 2015;36:2184-2196. 


\section{Cellular Physiology Cell Physiol Biochem 2016;38:1928-1938 \begin{tabular}{ll|l} 
DOI: 10.1159/000445554 & O 2016 The Author(s). Published by S. Karger AG, Basel \\
and Biochemisarger.com/cpb
\end{tabular} \\ Cheng et al.: Celastrol Ameliorates Cardiac Fibrosis and Dysfunction}

24 Chu W, Li X, Li C, Wan L, Shi H, Song X, Liu X, Chen X, Zhang C, Shan H, Lu Y, Yang B: TGFBR3, a potential negative regulator of TGF-beta signaling, protects cardiac fibroblasts from hypoxia-induced apoptosis. J Cell Physiol 2011;226:2586-2594.

25 Heldin CH, Landstrom M, Moustakas A: Mechanism of TGF-beta signaling to growth arrest, apoptosis, and epithelial-mesenchymal transition. Curr Opin Cell Biol 2009;21:166-176.

26 Zeisberg EM, Tarnavski O, Zeisberg M, Dorfman AL, McMullen JR, Gustafsson E, Chandraker A, Yuan X, Pu WT, Roberts AB, Neilson EG, Sayegh MH, Izumo S, Kalluri R: Endothelial-to-mesenchymal transition contributes to cardiac fibrosis. Nat Med 2007;13:952-961.

27 Schiller M, Javelaud D, Mauviel A: TGF-beta-induced SMAD signaling and gene regulation: consequences for extracellular matrix remodeling and wound healing. J Dermatol Sci 2004;35:83-92.

28 Kumarswamy R, Volkmann I, Jazbutyte V, Dangwal S, Park DH, Thum T: Transforming growth factor-betainduced endothelial-to-mesenchymal transition is partly mediated by microRNA-21. Arterioscler Thromb Vasc Biol 2012;32:361-369.

29 Liang H, Zhang C, Ban T, Liu Y, Mei L, Piao X, Zhao D, Lu Y, Chu W, Yang B: A novel reciprocal loop between microRNA-21 and TGFbetaRIII is involved in cardiac fibrosis. Int J Biochem Cell Biol 2012;44:2152-2160. 